Article

\title{
On $q$-Hermite-Hadamard Inequalities for Differentiable Convex Functions
}

\author{
Seksan Jhanthanam ${ }^{1}$, Jessada Tariboon ${ }^{2} \mathbb{D}$, Sotiris K. Ntouyas ${ }^{3,4}{ }^{(\mathbb{D}}$ and Kamsing Nonlaopon $1, * \mathbb{C}$ \\ 1 Department of Mathematics, Faculty of Science, Khon Kaen University, Khon Kaen 40002, Thailand \\ 2 Department of Mathematics, Faculty of Applied Science, King Mongkut's University of Technology North \\ Bangkok, Bangkok 10800, Thailand \\ 3 Department of Mathematics, University of Ioannina, 45110 Ioannina, Greece \\ 4 Nonlinear Analysis and Applied Mathematics (NAAM)-Research Group, Department of Mathematics, \\ Faculty of Science, King Abdulaziz University, P.O. Box 80203, Jeddah 21589, Saudi Arabia \\ * Correspondence: nkamsi@kku.ac.th; Tel.: +668-6642-1582
}

Received: 12 June 2019; Accepted: 15 July 2019; Published: 17 July 2019

\begin{abstract}
In this paper, we establish some new results on the left-hand side of the $q$-Hermite-Hadamard inequality for differentiable convex functions with a critical point. Our work extends the results of Alp et. al ( $q$-Hermite Hadamard inequalities and quantum estimates for midpoint type inequalities via convex and quasi-convex functions, J. King Saud Univ. Sci., 2018, 30, 193-203), by considering the critical point-type inequalities.
\end{abstract}

Keywords: Hermite-Hadamard inequalities; $q$-derivative; $q$-integral; convex functions

\section{Introduction}

Quantum calculus (also known as $q$-calculus) is the study of calculus without limits, where classical mathematical formulas are obtained as $q \rightarrow 1$. Firstly introduced by Euler (1707-1783) in the tracks of Newton's infinite series, the study of $q$-calculus was established in the early Twentieth Century after the work of Jackson (1910) on defining an integral later known as the $q$-Jackson integral; see [1-4]. In $q$-calculus, the classical derivative is replaced by the $q$-difference operator in order to deal with non-differentiable functions; see [5,6] for more details. Applications of $q$-calculus can be found in various fields of mathematics and physics, and the interested readers are referred to [7-10].

The theory of convex functions has been widely studied and applied to various fields of science. Due to its close relation to the theory of inequalities, a rich literature on inequalities can be found in the study of convex functions; see [11-18]. This includes the Hermite-Hadamard inequality, introduced by Hermite and Hadamard independently, which has been studied extensively in recent years.

Let $J \subseteq \mathbb{R}$ be an interval and $f: J \rightarrow \mathbb{R}$ be a function from $J$ to $\mathbb{R}$. Recall that $f$ is said to be a convex function if it satisfies the inequality:

$$
f(\lambda x+(1-\lambda) y) \leq \lambda f(x)+(1-\lambda) f(y)
$$

for all $x, y \in J$ and $\lambda \in[0,1]$. In addition, if an equality holds for all $x, y \in J$ and $\lambda \in[0,1]$, then $f$ is said to be affine.

It is also well known that $f$ is convex if and only if it satisfies the Hermite-Hadamard inequality, which is defined by:

$$
f\left(\frac{a+b}{2}\right) \leq \frac{1}{b-a} \int_{a}^{b} f(x) d x \leq \frac{f(a)+f(b)}{2}
$$


for all $a, b \in J$ and $a<b$. One can estimate by the right-hand side of (2) by using Iyengar's inequality, which is defined by:

$$
\left|\frac{f(a)+f(b)}{2}-\frac{1}{b-a} \int_{a}^{b} f(x) d x\right| \leq \frac{M(b-a)}{4}-\frac{1}{4 M(b-a)}(f(b)-f(a))^{2},
$$

where $M$ denotes the Lipschitz constant, that is $M=\sup \left\{\left|\frac{f(x)-f(y)}{x-y}\right| ; x \neq y\right\}$.

This fundamental result of Hermite and Hadamard has attracted many mathematicians, and consequently, this inequality has been generalized and extended in many directions; see [19-31] and the references cited therein.

In 2018, Alp et al. [32] studied the $q$-analogue of Hermite-Hadamard's inequality for increasing functions, that is,

$$
f\left(\frac{q a+b}{1+q}\right) \leq \frac{1}{b-a} \int_{a}^{b} f(x)_{a} d_{q} x \leq \frac{q f(a)+f(b)}{1+q},
$$

where $q$ is a constant with $0<q<1$. Moreover, they studied the generalized $q$-Hermite-Hadamard inequality for differentiable convex functions, that is,

$$
\max \left\{I_{1}, I_{2}, I_{3}\right\} \leq \frac{1}{b-a} \int_{a}^{b} f(x){ }_{a} d_{q} x \leq \frac{q f(a)+f(b)}{1+q},
$$

where:

$$
\begin{aligned}
& I_{1}=f\left(\frac{q a+b}{1+q}\right), \\
& I_{2}=f\left(\frac{a+q b}{1+q}\right)+\frac{(1-q)(b-a)}{1+q} f^{\prime}\left(\frac{a+q b}{1+q}\right), \\
& I_{3}=f\left(\frac{a+b}{2}\right)+\frac{(1-q)(b-a)}{2(1+q)} f^{\prime}\left(\frac{a+b}{2}\right) .
\end{aligned}
$$

This paper aims to establish the generalized $q$-Hermite-Hadamard inequality for differentiable convex functions with a critical point.

The paper is organized as follows. Some basic concepts are recalled in Section 2. Section 3 contain the main results, while conclusions are given in Section 4.

\section{Preliminaries}

In this section, some basic results are mentioned. Throughout this section, we let $J=[a, b] \subseteq \mathbb{R}$ be an interval and $q$ be a constant with $0<q<1$.

Definition 1. [33] The q-derivative of a continuous function $f: J \rightarrow \mathbb{R}$ at $x$ is defined as:

$$
{ }_{a} D_{q} f(x)=\frac{f(x)-f(q x+(1-q) a)}{(1-q)(x-a)}, \text { for } \quad x \neq a .
$$

For $x=a$, we define ${ }_{a} D_{q} f(a)=\lim _{x \rightarrow a} D_{q} f(x)$.

If ${ }_{a} D_{q} f(x)$ exists for all $x \in J$, then $f$ is $q$-differentiable on $J$. Moreover, if $a=0$, then (5) reduces to ${ }_{0} D_{q} f=D_{q} f$, where $D_{q}$ is the $q$-derivative of $f$, which is defined by:

$$
D_{q} f(x)=\frac{f(x)-f(q x)}{(1-q) x} .
$$

For more details, see [4].

The higher-order $q$-derivatives of functions on $J$ are also defined. 
Definition 2. [33] For a continuous function $f: J \rightarrow \mathbb{R}$, the second-order q-derivative of $f$ on $J$, if ${ }_{a} D_{q} f$ is $q$-differentiable on $J$, denoted by ${ }_{a} D_{q}^{2} f$ and defined by:

$$
{ }_{a} D_{q}^{2} f={ }_{a} D_{q}\left({ }_{a} D_{q}\right) .
$$

Similarly, provided that ${ }_{a} D_{q}^{n-1} f$ is the $q$-derivative on J for some integer $n>2$, the $n^{\text {th }}$-order $q$-derivative of $f$ on $J$ is the function from $J \rightarrow \mathbb{R}$ defined by:

$$
{ }_{a} D_{q}^{n} f={ }_{a} D_{q}\left(a D_{q}^{n-1} f\right) .
$$

Example 1. Let $f: J \rightarrow \mathbb{R}$ with $f(x)=x^{2}+1$. Let $q$ be a constant with $0<q<1$. Then, for $x \neq a$, we have:

$$
\begin{aligned}
{ }_{a} D_{q}\left(x^{2}+1\right) & =\frac{\left(x^{2}+1\right)-\left[(q x+(1-q) a)^{2}+1\right]}{(1-q)(x-a)} \\
& =\frac{(1+q) x^{2}-2 q a x-(1-q) a^{2}}{(x-a)} \\
& =(1+q) x+(1-q) a .
\end{aligned}
$$

For $x=a,{ }_{a} D_{q} f(a)=\lim _{x \rightarrow a}{ }_{a} D_{q} f(x)=2 a$.

Definition 3. [33] The q-integral of a continuous function $f: J \rightarrow \mathbb{R}$ is defined as:

$$
\int_{a}^{x} f(t){ }_{a} d_{q} t=(1-q)(x-a) \sum_{n=0}^{\infty} q^{n} f\left(q^{n} x+\left(1-q^{n}\right) a\right),
$$

for $x \in J$.

Note that if $a=0$, then (7) becomes the classical q-integral of $f$, that is,

$$
\int_{0}^{x} f(t){ }_{0} d_{q} t=(1-q) x \sum_{n=0}^{\infty} q^{n} f\left(q^{n} x\right)
$$

for $x \in[0, \infty)$; see [4] for more details.

Example 2. Let $f:[a, b] \rightarrow \mathbb{R}$ with $f(x)=2 x$. Let $q$ be a constant with $0<q<1$. Then, we have:

$$
\begin{aligned}
\int_{a}^{b} f(x){ }_{a} d_{q} x & =\int_{a}^{b} 2 x_{a} d_{q} x \\
& =2(1-q)(b-a) \sum_{n=0}^{\infty} q^{n}\left(q^{n} b+\left(1-q^{n}\right) a\right) \\
& =\frac{2(b-a)(b+q a)}{1+q} .
\end{aligned}
$$

Note that if $q \rightarrow 1$, we obtain the classical integration:

$$
\int_{a}^{b} f(x) d x=\int_{a}^{b} 2 x d x=b^{2}-a^{2} .
$$

Theorem 1. Assume that the function $f: J \rightarrow \mathbb{R}$ is continuous. Then, we have the following:

(i) ${ }_{a} D_{q} \int_{a}^{x} f(t){ }_{a} d_{q} t=f(x)-f(a)$;

(ii) $\int_{c}^{x}{ }_{a} D_{q} f(t){ }_{a} d_{q} t=f(x)-f(c)$ for $c \in(a, x)$.

Theorem 2. Assume that the functions $f, g: J \rightarrow \mathbb{R}$ are continuous and $\alpha \in \mathbb{R}$. Then, we have the following:

(i) $\int_{a}^{x}[f(t)+g(t)]_{a} d_{q} t=\int_{a}^{x} f(t){ }_{a} d_{q} t+\int_{a}^{x} g(t){ }_{a} d_{q} t$;

(ii) $\int_{a}^{x}(\alpha f)(t){ }_{a} d_{q} t=\alpha \int_{a}^{x} f(t){ }_{a} d_{q} t$; 
(iii) $\int_{c}^{x} f(t){ }_{a} D_{q} g(t){ }_{a} d_{q} t=\left.(f g)\right|_{c} ^{x}-\int_{c}^{x} g(q t+(1-q) a)_{a} D_{q} f(t){ }_{a} d_{q} t$ for $c \in(a, x)$.

For the proofs of the properties in Theorems 1 and 2, see [34].

\section{Main Results}

In this section, we present our main results on the left-hand side of the $q$-Hermite-Hadamard inequality for differentiable convex functions with a critical point.

Theorem 3. Suppose that $f:[a, b] \rightarrow \mathbb{R}$ is a differentiable convex function on $(a, b)$ such that $f^{\prime}(c)=0$ for $c \in(a, b)$, and let $q$ be a constant with $0<q<1$. Then, we have:

$$
\begin{aligned}
f\left(\frac{q(a+c)+(1-q) b}{1+q}\right)+ & f^{\prime}\left(\frac{q(a+c)+(1-q) b}{1+q}\right)\left(\frac{q(b-c)}{1+q}\right) \\
& \leq \frac{1}{b-a} \int_{a}^{b} f(x)_{a} d_{q} x \\
& \leq \frac{q f(a)+f(b)}{1+q}
\end{aligned}
$$

Proof. Since the function $f$ is differentiable on $(a, b)$, there exists a tangent line at the point $\frac{q(a+c)+(1-q) b}{1+q} \in(a, b)$, given by:

$$
h(x)=f\left(\frac{q(a+c)+(1-q) b}{1+q}\right)+f^{\prime}\left(\frac{q(a+c)+(1-q) b}{1+q}\right)\left(x-\frac{q(a+c)+(1-q) b}{1+q}\right) .
$$

Since $f$ is a convex function on $[a, b]$, it follows that $h(x) \leq f(x)$ for all $x \in[a, b]$. After $q$-integrating of (9) on $[a, b]$, we have:

$$
\begin{array}{rl}
\int_{a}^{b} & h(x){ }_{a} d_{q} x \\
= & \int_{a}^{b}\left[f\left(\frac{q(a+c)+(1-q) b}{1+q}\right)+f^{\prime}\left(\frac{q(a+c)+(1-q) b}{1+q}\right)\right. \\
& \left.\times\left(x-\frac{q(a+c)+(1-q) b}{1+q}\right)\right]{ }_{a} d_{q} x \\
= & (b-a) f\left(\frac{q(a+c)+(1-q) b}{1+q}\right) \\
& +f^{\prime}\left(\frac{q(a+c)+(1-q) b}{1+q}\right)\left(\int_{a}^{b} x_{a} d_{q} x-(b-a) \frac{q(a+c)+(1-q) b}{1+q}\right) \\
= & (b-a) f\left(\frac{q(a+c)+(1-q) b}{1+q}\right) \\
& +f^{\prime}\left(\frac{q(a+c)+(1-q) b}{1+q}\right)\left((b-a) \frac{(q a+b)}{1+q}-(b-a) \frac{q(a+c)+(1-q) b}{1+q}\right) \\
= & (b-a)\left[f\left(\frac{q(a+c)+(1-q) b}{1+q}\right)+f^{\prime}\left(\frac{q(a+c)+(1-q) b}{1+q}\right)\left(\frac{q(b-c)}{1+q}\right)\right] \\
\leq & \int_{a}^{b} f(x){ }_{a} d_{q} x .
\end{array}
$$

On the other hand, since $f$ is a convex function, we obtain:

$$
\begin{aligned}
\frac{1}{(b-a)} \int_{a}^{b} f(x)_{a} d_{q} x & =\frac{1}{(b-a)}\left[(1-q)(b-a) \sum_{n=0}^{\infty} q^{n} f\left(q^{n} b+\left(1-q^{n}\right) a\right)\right] \\
& =(1-q) \sum_{n=0}^{\infty} q^{n} f\left(q^{n} b+\left(1-q^{n}\right) a\right) \\
& \leq(1-q) \sum_{n=0}^{\infty} q^{n}\left[q^{n} f(b)+\left(1-q^{n}\right) f(a)\right] \\
& =(1-q)\left[\frac{f(b)}{1-q^{2}}+\frac{f(a)}{1-q}-\frac{f(a)}{1-q^{2}}\right] \\
& =\frac{q f(a)+f(b)}{1+q}
\end{aligned}
$$

The proof is complete. 
Remark 1. In Theorem 3 , if $q \in\left(0, \frac{c-b}{a-b}\right]$, then $\frac{q(a+c)+(1-q) b}{1+q} \in[c, b)$. We can reduce the left-hand side of Theorem 3 as:

$$
f\left(\frac{q(a+c)+(1-q) b}{1+q}\right) \leq \frac{1}{(b-a)} \int_{a}^{b} f(x){ }_{a} d_{q} x \leq \frac{q f(a)+f(b)}{1+q}
$$

since $f^{\prime}\left(\frac{q(a+c)+(1-q) b}{1+q}\right)\left(\frac{q(b-c)}{1+q}\right) \geq 0$.

Remark 2. In Remark 1, if $c \rightarrow a^{+}$, then $\frac{c-b}{a-b} \rightarrow 1^{-}$. Since $q \in(0,1)$, we have $\frac{q(a+c)+(1-q) b}{1+q} \in(a, b)$. We can reduce the left-hand side of Theorem 3 as:

$$
f(q a+(1-q) b) \leq \frac{1}{(b-a)} \int_{a}^{b} f(x){ }_{a} d_{q} x \leq \frac{q f(a)+f(b)}{1+q}
$$

since $f^{\prime}\left(\frac{q(a+c)+(1-q) b}{1+q}\right)\left(\frac{q(b-c)}{1+q}\right) \geq 0$.

Corollary 1. Assume that $f:[a, b] \rightarrow \mathbb{R}$ is a differentiable convex function on $(a, b)$ such that $f^{\prime}\left(\frac{a+b}{2}\right)=0$, for $0<q<1$. Then, we have:

$$
\begin{aligned}
f\left(\frac{q\left(a+\frac{a+b}{2}\right)+(1-q) b}{1+q}\right) & +f^{\prime}\left(\frac{q\left(a+\frac{a+b}{2}\right)+(1-q) b}{1+q}\right) \frac{q(b-a)}{2(1+q)} \\
& \leq \frac{1}{b-a} \int_{a}^{b} f(x)_{a} d_{q} x \\
& \leq \frac{q f(a)+f(b)}{1+q} .
\end{aligned}
$$

Corollary 2. Assume that $f:[a, b] \rightarrow \mathbb{R}$ is a differentiable convex function on $(a, b)$ such that $f^{\prime}(0)=0$, for $0 \in(a, b)$ and $0<q<1$. Then, we have:

$$
\begin{aligned}
f\left(\frac{q a+(1-q) b}{1+q}\right) & +f^{\prime}\left(\frac{q a+(1-q) b}{1+q}\right) \frac{q b}{(1+q)} \\
& \leq \frac{1}{b-a} \int_{a}^{b} f(x)_{a} d_{q} x \leq \frac{q f(a)+f(b)}{1+q} .
\end{aligned}
$$

Theorem 4. Let $f:[a, b] \rightarrow \mathbb{R}$ be a differentiable convex function on $(a, b)$ such that $f^{\prime}(c)=0$ for $c \in(a, b)$ and $0<q<1$. Then, we have:

$$
\begin{aligned}
f\left(\frac{(1-q) a+q(c+b)}{1+q}\right) & +f^{\prime}\left(\frac{(1-q) a+q(c+b)}{1+q}\right)\left(\frac{q(2 a-b-c)+b-a}{1+q}\right) \\
& \leq \frac{1}{b-a} \int_{a}^{b} f(x){ }_{a} d_{q} x \\
& \leq \frac{q f(a)+f(b)}{1+q}
\end{aligned}
$$

Proof. Since the function $f$ is differentiable on $(a, b)$, there exists a tangent line at the point $\frac{(1-q) a+q(c+b)}{1+q} \in(a, b)$, which is given by:

$$
k(x)=f\left(\frac{(1-q) a+q(c+b)}{1+q}\right)+f^{\prime}\left(\frac{(1-q) a+q(c+b)}{1+q}\right)\left(x-\frac{(1-q) a+q(c+b)}{1+q}\right) .
$$


Since $f$ is convex on $[a, b]$, it follows that $k(x) \leq f(x)$ for all $x \in[a, b]$. After $q$-integrating (13), we obtain:

$$
\begin{aligned}
& \int_{a}^{b} k(x){ }_{a} d_{q} x \\
& =\int_{a}^{b}\left[f\left(\frac{(1-q) a+q(c+b)}{1+q}\right)\right. \\
& \left.\quad+f^{\prime}\left(\frac{(1-q) a+q(c+b)}{1+q}\right)\left(x-\frac{(1-q) a+q(c+b)}{1+q}\right)\right]{ }_{a} d_{q} x \\
& =(b-a) f\left(\frac{(1-q) a+q(c+b)}{1+q}\right) \\
& \quad+f^{\prime}\left(\frac{1-q) a+q(c+b)}{1+q}\right)\left(\int_{a}^{b} x_{a} d_{q} x-(b-a) \frac{(1-q) a+q(c+b)}{1+q}\right) \\
& =(b-a) f\left(\frac{(1-q) a+q(c+b)}{1+q}\right) \\
& \quad+f^{\prime}\left(\frac{(1-q) a+q(c+b)}{1+q}\right)\left[(b-a)\left(\left(\frac{a q+b}{1+q}\right)-\frac{(1-q) a+q(c+b)}{1+q}\right)\right] \\
& =(b-a)\left[f\left(\frac{(1-q) a+q(c+b)}{1+q}\right)\right. \\
& \left.\quad+f^{\prime}\left(\frac{(1-q) a+q(c+b)}{1+q}\right)\left(\frac{q(2 a-b-c)+b-a}{1+q}\right)\right] \\
& \leq \int_{a}^{b} f(x)_{a} d_{q} x .
\end{aligned}
$$

The proof is complete.

Remark 3. In Theorem 4, if $q \in\left(\frac{1}{2}, \frac{c-a}{b-a}\right]$, then $f^{\prime}\left(\frac{(1-q) a+q(c+b)}{1+q}\right) \leq 0$ and $\frac{q(2 a-b-c)+b-a}{1+q}<0$. We can reduce the left-hand side of Theorem 4 as:

$$
f\left(\frac{(1-q) a+q(c+b)}{1+q}\right) \leq \frac{1}{(b-a)} \int_{a}^{b} f(x){ }_{a} d_{q} x \leq \frac{q f(a)+f(b)}{1+q}
$$

since $f^{\prime}\left(\frac{(1-q) a+q(c+b)}{1+q}\right)\left(\frac{q(2 a-b-c)+b-a}{1+q}\right) \geq 0$

Remark 4. In Remark 3, if $c \rightarrow b^{-}$, then $q \rightarrow 1^{-}$. Since $q \in\left(\frac{1}{2}, 1\right)$, we have $\frac{(1-q) a+q(b+c)}{1+q} \in\left(\frac{a+2 b}{3}, b\right)$. We can reduce the left-hand side of Theorem 4 as:

$$
\left.f\left((1-q)\left(\frac{2 a+b}{3}\right)+q b\right)\right) \leq \frac{1}{(b-a)} \int_{a}^{b} f(x)_{a} d_{q} x \leq \frac{q f(a)+f(b)}{1+q}
$$

since $f^{\prime}\left(\frac{(1-q) a+q(c+b)}{1+q}\right)\left(\frac{q(2 a-b-c)+b-a}{1+q}\right) \geq 0$.

Theorem 5. [Generalized q-Hermite-Hadamard inequality for convex differentiable functions]. Let $f:[a, b] \rightarrow$ $\mathbb{R}$ be a differentiable convex function on $(a, b)$ such that $f^{\prime}(c)=0$ for $c \in(a, b)$ and $0<q<1$. Then, we have:

$$
\max \left\{I_{1}, I_{2},\right\} \leq \frac{1}{b-a} \int_{a}^{b} f(x){ }_{a} d_{q} x \leq \frac{q f(a)+f(b)}{1+q},
$$

where

$$
\begin{aligned}
& I_{1}=f\left(\frac{q(a+c)+(1-q) b}{1+q}\right)+f^{\prime}\left(\frac{q(a+c)+(1-q) b}{1+q}\right)\left(\frac{q(b-c)}{1+q}\right), \\
& I_{2}=f\left(\frac{(1-q) a+q(c+b)}{1+q}\right)+f^{\prime}\left(\frac{(1-q) a+q(c+b)}{1+q}\right) \\
& \left(\frac{q(2 a-b-c)+b-a}{1+q}\right) .
\end{aligned}
$$

Proof. A combination of (8) and (12) yields (15). Thus, the proof is complete. 
Example 3. Define the function $f(x)=x^{2}$ on $[-1,3]$, and let $q \in(0,1)$. Applying Theorem 3 with $a=-1$, $b=3$, and $c=0$, the left-hand side becomes:

$$
\begin{aligned}
& f\left(\frac{q(a+c)+(1-q) b}{1+q}\right)+f^{\prime}\left(\frac{q(a+c)+(1-q) b}{1+q}\right) \frac{q(b-c)}{1+q}-\frac{1}{b-a} \int_{a}^{b} f(x)_{a} d_{q} x \\
& \quad=f\left(\frac{3-4 q}{1+q}\right)+f^{\prime}\left(\frac{3-4 q}{1+q}\right)\left(\frac{3 q}{1+q}\right)-\frac{1}{4}\left[4(1-q) \sum_{n=0}^{\infty} q^{n} f\left(3 q^{n}-\left(1-q^{n}\right)\right)\right] \\
& \quad=\frac{-9 q^{4}-9 q^{3}-9 q^{2}-16 q}{(1+q)^{2}\left(1+q+q^{2}\right)} \leq 0 .
\end{aligned}
$$

For the right-hand side, we have:

$$
\frac{1}{3-(-1)} \int_{-1}^{3} x^{2}{ }_{a} d_{q} x-\frac{q f(-1)+f(3)}{1+q}=\frac{16}{1+q+q^{2}}-\frac{8}{1+q}+1-\frac{9+q}{1+q} \leq 0 .
$$

Example 4. Define function $f(x)=x^{2}$ on $[-1,1]$, and let $q \in(0,1)$. Applying Corollary 2 with $a, b=-1$ and $c=0$, the left hand-side becomes:

$$
\begin{gathered}
f\left(\frac{q a+(1-q) b}{1+q}\right)+f^{\prime}\left(\frac{q a+(1-q) b}{1+q}\right) \frac{(q b)}{1+q}-\frac{1}{b-a} \int_{a}^{b} f(x)_{a} d_{q} x \\
=f\left(\frac{1-2 q}{1+q}\right)+f^{\prime}\left(\frac{1-2 q}{1+q}\right)\left(\frac{q}{1+q}\right)-(1-q) \sum_{n=0}^{\infty} q^{n} f\left(2 q^{n}-1\right) \\
=\frac{4 q^{2}-4 q+1}{(1+q)^{2}}+\frac{2 q(1-2 q)}{(1+q)^{2}}-\frac{1+2 q-2 q^{2}+q^{3}}{\left(1+q+q^{2}\right)(1+q)} \leq 0 .
\end{gathered}
$$

For the right-hand side, we have:

$$
\begin{aligned}
\frac{1}{1-(-1)} \int_{-1}^{1} x_{a}^{2} d_{q} x & -\frac{q f(-1)+f(1)}{1+q} \\
& =\frac{1}{2}\left[(1-q)(2) \sum_{n=0}^{\infty} q^{n} f\left(q^{n}-\left(1-q^{n}\right)\right)\right]-\frac{1+q}{1+q} \\
& =\frac{4}{1+q+q^{2}}-\frac{4}{1+q}+1-1 \leq 0 .
\end{aligned}
$$

Example 5. Define functions $f(x)=x^{2}$ on $[-3,1]$, and let $q \in(0,1)$. Applying Theorem 4 with $a=-3$, $b=1$, and $c=0$, the left-hand side becomes:

$$
\begin{aligned}
f & \left(\frac{(1-q) a+q(c+b)}{1+q}\right)+f^{\prime}\left(\frac{(1-q) a+q(c+b)}{1+q}\right)\left(\frac{q(2 a-b-c)+b-a}{1+q}\right) \\
& -\frac{1}{b-a} \int_{a}^{b} f(x)_{a} d_{q} x \\
= & f\left(\frac{4 q-3}{1+q}\right)+f^{\prime}\left(\frac{4 q-3}{1+q}\right)\left(\frac{4-7 q}{1+q}\right)-\frac{1}{4}\left[4(1-q) \sum_{n=0}^{\infty} q^{n} f\left(4 q^{n}-3\right)\right] \\
= & \frac{16 q^{2}-24 q+9}{(1+q)^{2}}+\frac{-56 q^{2}+74 q-24}{(1+q)^{2}}-\frac{16}{1+q+q^{2}}+\frac{24}{1+q}-9 \leq 0 .
\end{aligned}
$$


For the right-hand side, we have:

$$
\frac{1}{3-(-1)} \int_{-1}^{3} x^{2}{ }_{a} d_{q} x-\frac{q f(-3)+f(1)}{1+q}=\frac{16}{1+q+q^{2}}-\frac{24}{1+q}+9-\frac{9 q+1}{1+q} \leq 0 .
$$

\section{Conclusions}

In this paper, we considered and investigated the class of differentiable convex functions, which has a critical point in the setting of $q$-calculus. We used the approach of $q$-calculus to derive some new results on the left-hand side of $q$-Hermite-Hadamard inequalities. It is expected that the ideas and techniques presented in this paper will stimulate further research in this field.

Author Contributions: All authors contributed equally to this article. They read and approved the final manuscript. Funding: This research received no external funding.

Acknowledgments: The fourth author was financially supported by the National Research Council of Thailand and Research Fund, Faculty of Science, Khon Kaen University.

Conflicts of Interest: The authors declare no conflict of interest.

\section{References}

1. Brito, A.M.C.; Cruz, D.A. Symmetric Quantum Calculus. Ph.D. Thesis, Aveiro University, Aveiro, Portugal, 2012.

2. Ernst, T. The History of $q$-Calculus and a New Method; UUDM Report 2000:16; Department of Mathematics, Uppsala University: Uppsala, Sweden, 2000.

3. Jackson, F.H. On a q-definite integrals. Q. J. Pure Appl. Math. 1910, 41, 193-203.

4. Kac, V.; Cheung, P. Quantum Calculus; Springer: New York, NY, USA, 2002.

5. Almeida, R.; Torres, D.F.M. Non-differentiable variational principles in terms of a quantum operator. Math. Methods Appl. Sci. 2011, 34, 2231-2241.

6. Cresson, J.; Frederico, G.S.F.; Torres, D.F.M. Constants of motion for Non-differentiable quantum variational problems. Topol. Methods Nonlinear Anal. 2009, 33, 217-231. [CrossRef]

7. Askey, R.; Wilson, J. Some basic hypergeometric orthogonal polynomials that generalize the Jacobi polynomails. Mem. Am. Math. Soc. 1985, 54, 1-55.

8. Ismail, M.E.H.; Simeonov, P. $q$-Difference operators for orthogonal polynomials. J. Comput. Appl. Math. 2009, 233, 749-761. [CrossRef]

9. Page, D.N. Information in black hole radiation. Phys. Rev. Lett. 1993, 71, 3743-3746. [CrossRef] [PubMed]

10. Youm, D. q-deformed conformal quantum mechanics. Phys. Rev. D 2000, 62, 095009. [CrossRef]

11. Taf, S.; Brahim, K.; Riahi, L. Some results for Hadamard-type inequalities in quantum calculus. Le Matematiche 2014, 69, 243-258.

12. Dragomir, S.S. Two refinements of Hadamard's inequalities. Coll. Sci. Pap. Fac. Kragujevac 1990, 11, 23-26.

13. Dragomir, S.S. Some integral inequalities for differentiable convex functions. Contrib. Sec. Math. Tech. Sci. 1992, 13, 13-17.

14. Noor, M.A.; Noor, K.I.; Awan, M.U. Generalized convexity and integral inequalities. Appl. Math. Inf. Sci. 2005, 9, 233-243. [CrossRef]

15. Noor, M.A.; Noor, K.I.; Awan, M.U. Some quantum estimates for Hermite-Hadamard inequalities. Appl. Math. Comput. 2015, 251, 675-679. [CrossRef]

16. Noor, M.A.; Noor, K.I.; Awan, M.U. Quantum ostrowski inequalities for $q$-differentiable convex functions. J. Math. Inequal. 2016, 10, 1013-1018. [CrossRef]

17. Wang, W.; Qi, J. Some new estimates of Hermite-Hadamard convex functions with applications. Int. J. Anal. Appl. 2017, 13, 15-21.

18. Prabseang, J.; Nonlaopon, K.; Tariboon, J. Quantum Hermite-Hadamard inequalities for double integral and q-differentiable convex functions. J. Math. Inequal. 2019, 13, 675-686.

19. Cristescu, G. Improved integral inequalities for products of convex functions. J. Inequal. Pure Appl. Math. 2005, 6, Article 35 . 
20. Cristescu, G.; Noor, M.A.; Awan, M.U. Bounds of the second degree cumulative frontier gaps of functions with generalized convexity. Carpath. J. Math. 2015, 31, 173-180.

21. Dragomir, S.S.; Agarwal, R.P. Two inequalities for differentiable mappings and applications to special means of real numbers and to trapezoidal formula. Appl. Math. Lett. 1998, 11, 91-95. [CrossRef]

22. Dragomir, S.S.; Pearce, C.E.M. Selected Topics on Hermite-Hadamard Inequalities and Applications; Victoria University: Footscray, Australia, 2000.

23. Ion, D.A. Some estimates on the Hermite-Hadamard inequality through quasi-convex functions. Ann. Univ. Craiova, Math. Comput. Sci. Ser. 2007, 34, 82-87.

24. Khattri, S.K. Three proofs of the inequality $e<\left(1+\frac{1}{2}\right)^{n+0.5}$. Am. Math. Mon. 2010, 17, $273-277$.

25. Niculescu, C.P.; Persson, L.-E. Convex Functions and Their Applications; Springer: New York, NY, USA, 2006.

26. Noor, M.A. Hermite-Hadamard integral inequalities for log -preinvex functions. J. Math. Anal. Approx. 2007, 2, 126-131.

27. Noor, M.A. On Hermite-Hadamard integral inequalities for involving two log -preinvex functions. J. Inequal. Pure Appl. Math. 2007, 3, 75-81.

28. Noor, M.A. Hadamard integral inequalities for product of two preinvex functions. Nonlinear Anal. 2009, 14, 167-173.

29. Noor, M.A.; Awan, M.U.; Noor, K.I. On some inequalities for relative semi-convex functions. J. Inequal. Appl. 2013, 2013, 332. [CrossRef]

30. Noor, M.A.; Noor, K.I.; Awan, M.U. Quantum analogues of Hermite-Hadamard type inequalities for generalized convexity. In Computation, Cryptography and Network Security; Daras, N., Rassias, M.T., Eds.; Springer: Cham, Switzerland, 2015.

31. Noor, M.A.; Noor, K.I.; Awan, M.U.; Li, J. On Hermite-Hadamard inequalities for h-preinvex functions. Filomat 2014, 28, 1463-1474. [CrossRef]

32. Alp, N.; Sarıkaya, M.Z.; Kunt, M.; İşcan, İ. q-Hermite-Hadamard inequalities and quantum estimates for midpoint type inequalities via convex and quasi-convex functions. J. King Saud. Univ. Sci. 2018, 30, 193-203. [CrossRef]

33. Tariboon, J.; Ntouyas, S.K. Quantum integral inequalities on finite intervals. J. Inequal. Appl. 2014, $2014,121$. [CrossRef]

34. Tariboon, J.; Ntouyas, S.K. Quantum calculus on finite intervals and applications to impulsive difference equations. Adv. Difference Equ. 2013, 2013, 282. [CrossRef]

(C) 2019 by the authors. Licensee MDPI, Basel, Switzerland. This article is an open access article distributed under the terms and conditions of the Creative Commons Attribution (CC BY) license (http:/ / creativecommons.org/licenses/by/4.0/). 